\title{
Incidence of Helicobacter Pylori in upper gastrointestinal lesion
}

\author{
Kumar V. ${ }^{1}$, Nag B. P. ${ }^{2}$, Anand P. ${ }^{3}$, Joshi N. ${ }^{4}$, Jain R. ${ }^{5}$ \\ ${ }^{1}$ Dr. Vikas Kumar, Assistant Professor, Rama Medical College Hospital Kanpur, Uttar Pradesh, ${ }^{2}$ Dr. B. P. Nag, \\ Professor, Mahatma Gandhi Medical College, Jaipur, Rajasthan, ${ }^{3}$ Dr. Pallavi Anand, Associate Professor, Rama Medical \\ College, Kanpur, Uttar Pradesh, ${ }^{4}$ Dr. Narayani Joshi, HOD and Professor, Mahatma Gandhi Medical College Jaipur \\ Rajasthan, ${ }^{5}$ Dr. Renu Jain, HOD and Professor, Rama Medical College Kanpur, Uttar Pradesh, India.
}

Corresponding Author: Dr. Vikas Kumar, Assistant Professor, Rama Medical College Hospital Kanpur, Uttar Pradesh, Email: vikasks@hotmail.com

\begin{abstract}
Introduction: Helicobacter pylori are gram-negative bacillus. It is noninvasive and live in gastric mucus, with the small proportion of bacteria adherent to the mucosa. Its spiral shape and flagella render Helicobacter pylori motile in mucus environment. Helicobacter pylori is an important pathogen in human causing chronic gastritis and playing a major role in the development of peptic ulcers and gastric cancer. Material and Methods: Study was conducted in Mahatma Gandhi Medical College and hospital, Jaipur (from Jan 2014 to March 2015). 57 Gastrointestinal biopsies were taken who have symptom of dyspepsia. All biopsies taken from different sites were brought in $10 \%$ buffered formalin. After overnight fixation in formalin, the tissues were processed in automated tissue processor for dehydration, clearing, and paraffin embedding. Section were cut in rotary microtome at 4 micrometer thickness. The section was stained for Hematoxylin and Eosin stain and Geimsa overnight stain. Result: The present studyincluded 57 cases of upper GIT biopsies (gastro esophageal duodenal biopsies). The incidence of Helicobacter Pylori was seen with chronic gastritis in our study. Conclusion: H. pyloriare etiologically associated with chronic activegastritis, duodenal ulcer, gastric ulcer, primary gastric B-cell lymphoma or mucosal associated-lymphoid type lymphoma (MALT lymphoma) and gastric adenocarcinoma. Helicobacter pyloriinducedchronic gastritis were encountered in 16(47.22\%) casesofthe presentstudy. Overnight Giemsa stain was done on all the patients and a histopathological diagnosis was made along with the study of the incidence of Helicobacterpylori positivity in all these patients.
\end{abstract}

Keywords: H. Pylori, Biopsy, Endoscopy, Histopathology, Upper GI lesions.

\section{Introduction}

Helicobacter pylori is an important pathogen in human causing chronic gastritis and playing a major role in the development of peptic ulcers and gastric cancer [1]. Helicobacter pylori are gram-negative bacillus. It is noninvasive and lives in gastric mucus, with the small proportion of bacteria adherent to the mucosa. Its spiral shape and flagella render Helicobacter pylori motile in mucus environment [2].

Helicobacter pylori coloni-zation induces tissue response in the gastric mucosa causing Gastritis [2]. These gram-negative bacilli can be detected by different methods including invasive techniques like histological examination, culture, and rapid urease test. The Noninvasive techniques comprise serology, urea breath test

Manuscript received: $2^{\text {nd }}$ January 2018

Reviewed: $12^{\text {th }}$ January 2018

Author Corrected: $18^{\text {th }}$ January 2018

Accepted for Publication 22 $2^{\text {nd }}$ January 2018 in addition to urine, blood and stool examination [3]. Acute H. pylori infection does not produce sufficient symptoms to require medical attention in most cases; it is chronic gastritis that ultimately causes the individual to seek treatment. H. pylori organisms are present in $90 \%$ of individuals with chronic gastritis affecting the antrum.

In addition, H. pylori have important roles in other gastric and duodenal diseases. For example, the increased acid secretion that occurs in $\mathrm{H}$. pylori gastritis may result in peptic ulcer disease, and $H$. pylori infection also confers increased risk of gastric cancer [4].

Effective treatments for $\mathrm{H}$. pylori infection include combinations of antibiotics and proton pump inhibitors [4]. 


\section{Aims and Objectives}

1. To detect Helicobacter Pylori infection in the biopsy from the upper gastrointestinal tract.
2. To study the incidence of Helicobacter Pylori in a various pathological lesion of upper gastrointestinal tract.

\section{Material \& Method}

The study was conducted in Mahatma Gandhi Medical College and hospital, Jaipur (from Jan 2014 to March 2015). 57 Gastrointestinal biopsies were taken who have symptoms of dyspepsia.

Inclusion Criteria: The study will include all biopsy which will be done for various chronic upper abdominal symptoms-abdominal pain, dyspepsia, heartburn, nausea, vomiting, and also for associated systemic manifestations like anorexia, weight loss.

Exclusion Criteria: Acute symptoms, autolysed specimen.

Study Sample Design: Prospective Study

Bias: Selection Bias (Cases with Dyspepsia)

All endoscopic biopsies taken from different sites are brought in $10 \%$ buffered formalin. After overnight fixation in formalin, the tissue was processed in automated tissue processor for dehydration, clearing, and paraffin embedding.

Embedding: Impregnated tissue imbedded in paraffin wax in mold

Section were cut in rotary microtome at 4 micrometer thickness. The section was stained for Hematoxylin and Eosin stain and Geimsa overnight stain.

The density of H.pylori was graded as follows:

a) 0 (negative grade): H.pylori not detected(Absent)

b) 1+(grade 1): occasionalbacilli after an extensive search(Mild)

c) $2+($ grade2): H.pylori seen in fair numbers or occasional clusters per3-5 oil immersion fields in mucous layers (Moderate)

d) 3+(grade3): Numerous bacilli in groups and clusters present in almost all oil immersion fields in mucus layers.(severe)

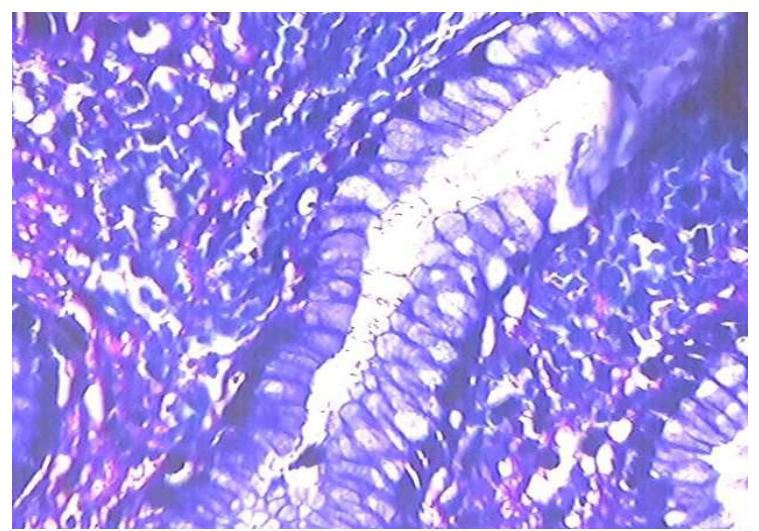

Figure 1: Helicobacter Pylori $3^{+}$in gastric mucosa (Giemsa stain, 100X)

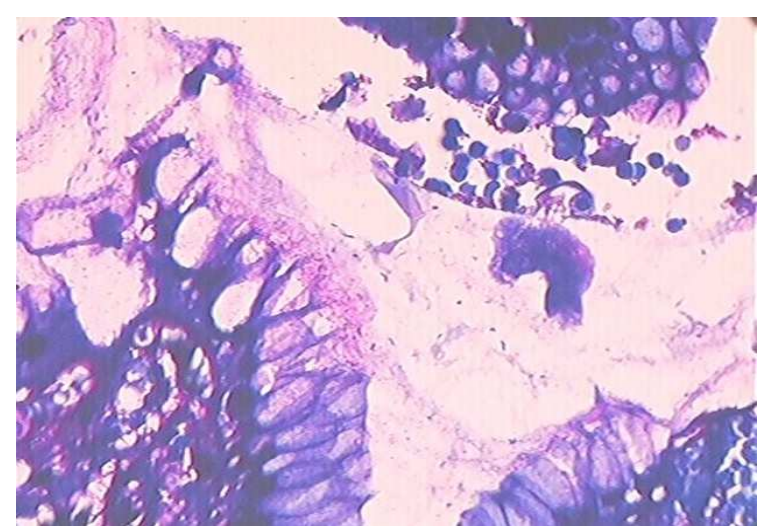

Figure 2: Helicobacter Pylori $2^{+}$in gastric mucosa (Giemsa stain, 100X) 


\section{Results}

Table 1: Site wise distribution of all upper gastrointestinal lesion

\begin{tabular}{|c|c|c|}
\hline Lesion & Total & Percentage \\
\hline Esophageal lesion & 7 & 12.28 \\
\hline Gastric lesion & 39 & 68.42 \\
\hline Duodenal lesion & 11 & 19.29 \\
\hline Total & $\mathbf{5 7}$ & $\mathbf{1 0 0}$ \\
\hline
\end{tabular}

The present study included 57 cases of upper GIT biopsies (gastro esophageal duodenal biopsies). H. Pylori infection is associated with chronic gastritis, Gastric ulcer.

In the study conducted among all the GIT biopsies, the maximum number of biopsies were from gastric lesion with $68.42 \%$, followed by duodenum with $19.29 \%$ and then esophageal biopsies whichwere $12.28 \%$.

Table-2: Age distribution of various lesion.

\begin{tabular}{|c|c|c|}
\hline $\begin{array}{c}\text { Age } \\
(\text { years })\end{array}$ & No of cases & \% \\
\hline$<20$ & 4 & 7.01 \\
\hline $20-29$ & 16 & 15.78 \\
\hline $30-39$ & 9 & 26.31 \\
\hline $40-49$ & 15 & 10.52 \\
\hline $50-59$ & 6 & 7.01 \\
\hline $60-69$ & 4 & 5.26 \\
\hline $70-80$ & 3 & $\mathbf{1 0 0}$ \\
\hline Total & $\mathbf{5 7}$ & \\
\hline
\end{tabular}

Most cases belong to the age group between 20-29 years and 40-49 years. In individual gastric lesion, site of distribution was antral, fundal, gastric body. An antral biopsy was taken in $84.6 \%$, fundal biopsy in $7.7 \%$ and gastric body biopsy in $7.7 \%$.

Table-3 showing incidence of $\mathbf{H}$. Pylori in gastric lesion.

\begin{tabular}{|c|c|c|}
\hline H. Pylori & No & \% \\
\hline Absent & 23 & 59.0 \\
\hline Mild 1+(grade 1) & 13 & 33.3 \\
\hline Moderate $+($ grade2) & 1 & 2.6 \\
\hline Severe 3+(grade3) & 2 & 5.1 \\
\hline Grand Total & $\mathbf{3 9}$ & $\mathbf{1 0 0 . 0}$ \\
\hline
\end{tabular}

H. pylori in 23 cases out of 39 (59\%) gastric biopsies. Mild Helicobacter pylori infection was seen in 13 cases (33.3\%). 1 case $(2.6 \%)$ show moderate infection. only 2 cases $(5.1 \%)$ show severe infection.

\section{Discussion}

All 57 cases which are included in the study were advised for biopsy of upper gastrointestinal tract (cases with dyspepsia).

Gastroscopy is a method of examination of the inside of gastrointestinal tract by using a thin flexible fiber-optic instrument called as the endoscope. It is currently the major method for diagnosis of gastrointestinal diseases. The number of gastro intestinal biopsies has increased after the invention of the flexible endoscope.Since the discovery of $\mathrm{H}$. pylori, it has been implicated in the pathogenesis of a number of gastrointestinal disorders including acute and chronic gastritis, gastric and duodenal ulceration and gastric cancers. 
H. pylori infection is the most common cause of chronic gastritis. The disease most often presents as a predominantly antral gastritis with high acid production, despite hypo gastrinemia. The risk of duodenal ulcer is increased in these patients and, in most; gastritis is limited to the antrum with occasional involvement of the cardia [4].

Although the mechanisms by which $\mathrm{H}$. pylori causes gastritis are incompletely defined, it is clear that infection results in increased acid production and disruption of normal gastric and duodenal protective mechanisms. H. pylori gastritis is, therefore, the result of an imbalance between gastroduodenal mucosal defenses and damaging forces that overcome those defenses [4]. Effective treatments for H. pylori infection include combinations of antibiotics and proton pump inhibitors [4].

Although, Although we found association of H.pylori with chronic duodenitis but in our study, there is no relation between $\mathrm{H}$. Pylori and chronic duodenitis.

In the present study, the male to female ratio was 0.96:1.(i.e. Males 28, and females 29). According to Sandhya Panjeta Gulia (2012) [5] et al out of 192 gastrointestinal endoscopic biopsies, 122(63.54\%) were males and $70(36.46 \%)$ were females; male: female ratio being $1.74: 1$

According to Mustapha et al (2007) [6] sex ratio was M: $\mathrm{F}=1.07: 1$, which shows the male predominance. The reason could be because a large number of male patients' attending the outpatient department of the hospital compared to the female patients.

In the present study the gastric lesions incidence of M: F ratio-0.85:1, In duodenal lesion it was $0.57: 1$ where asesophageallesion had a ratio of $6: 1$. Out of 57 patients, only 29 were females $(50.87 \%)$.

The incidence of gastric diseases: Among gastric lesions, commonest was chronic gastritis with $92.3 \%$ other gastric lesions were gastric carcinoma with the incidence of 7.6 .

Out of 192 gastrointestinal endoscopic biopsies, 168 comprised of inflammatory lesions, $3(1.54 \%)$ cases of chronic non-specific esophagi is, Barret esophagus $3(1.54 \%)$, GERD 3(1.54\%), all types of gastritis comprising 146(76.04\%) cases, gastric ulcer 7(3.59\%) and duodenitis $6(3.13 \%)$; one case $(0.51 \%)$ of premalignant lesion (esophageal dysplasia) and 12 cases
$(6.25 \%)$ of malignant lesions of esophagus and stomach. 10(5.62\%) cases had normal histology whereas one case was inadequate for an opinion [5].

A study conducted in Nigeria by Mustapha et al (2007) [6] showed lesions in descending order of their frequency of occurrence as reflux esophagitis, gastritis, gastric ulcer, duodenal ulcer \& gastric cancer.

But in study conducted endoscopic findings in 197 randomly selected subjects were gastritis- $89.7 \%$, peptic ulcer-6.6\%, gastric adenocarcinoma-1.01\% [7].

Thus, the prevalence of various upper GIT lesions varies among different studies. But gastritis predominates as the most common lesion as observed by us and the above authors.

Incidence of Helicobacter Pylori- H. pylori induced chronic gastritis we reencountered in $16(47.22 \%)$ cases of the present study. Giemsa stain demonstrated $\mathrm{H}$. pylori. Of the thirty-nine cases of chronic gastritis, twenty (51.28\%) cases showed activity. Among the 20 cases, eight (40\%) cases showed H. pylori positivity.

It is the most common infection in the world. In many developing countries the prevalence of $\mathrm{H}$. pylori close to $90 \%$ with very high percentages of infected children, suggesting exposure to the bacteria early in life. In established industrialized countries (western Europe, United States, Canada, and Australia), exposure occurs later, resulting in minimal percentages of infected children $(<1 \%$ in Swedish and Danish school children in the year 2000) and low percentages of infected adults (30\% by the age of 50 years) [8].

Gastritis: Among Thirty-six cases of chronic gastritis, sixteen cases $(41.02 \%)$ were found to be positive for $\mathrm{H}$. pylori in the present study. Kalebi A et al (2007) in their study on superficial gastritis showed H. pylori infection in antrum with neutrophilic infiltration in $91 \%$ of cases. ${ }^{43}$ The inflammatory infiltrate in $\mathrm{H}$. pylori associated gastritis is usually mixed mononuclear and neutrophilic [9]. In more severe cases intraepithelial neutrophils may be seen in the surface epithelium and in the gastric pits as micro abscesses. In our study, intraepithelial neutrophils were observed but none of the cases with active gastritis showed micro abscesses.

Wyatt JI and Rathbone BJ (1998) investigated 419 pairs of antral and corporal mucosal biopsy specimens and found lymphoid follicles in $27.4 \%$ of patients with $\mathrm{H}$ pylori associated gastritis [10]. 
In 1984, Marshall BJ and Warren JR in their study showed that among twenty cases of chronic gastritis, twelve were positive for H. pylori $(60 \%)$ [11].

In our study, the number of cases of $\mathrm{H}$. pylori increased as the severity of gastritis increased. Misra V et al (2007) in their study showed that the numbers of Helicobacter pylori positive cases were increased with the increasing grades of gastritis and the association were found to be statistically significant [12].

Of the thirty-nine cases of chronic gastritis, twenty $(51.28 \%)$ cases showed activity. Among the 20 cases, eight $(40 \%)$ cases showed H. pylori positivity.

Eidt S and Stolte M (1993) studied "two to three antral specimens" from 2692 patients with $\mathrm{H}$ pylori infection and detected lymphoid follicles and aggregates in 54\% of those patients [13].

Genta RM and Hammer HW (1994) found that the prevalence of lymphoid follicles was $63.8 \%$ (110 of 174 ) in patients with chronic gastritis and $100 \%$ in patients with $\mathrm{H}$ pylori infection [14].

Chen XY et al (2002) showed that lymphoid follicles and aggregates were found in the gastric antral mucosa of $76.0 \%$ (350 of 438 ) of patients with various $\mathrm{H}$ pylori associated gastric diseases [15]. In our study, similar to other studies, among four, three $(75 \%)$ cases with lymphoid aggregates or lymphoid follicles showed H.pylori positivity. The lymphoma association has proven to be particularly intriguing, because there are increasing numbers of reports of regression of lymphoma and lymphoid infiltrate after successful eradication of $H$. pylori infection [16]. However, there were no cases of gastric lymphoma in the present study.

Intestinal Metaplasia: Atrophic gastritis and intestinal metaplasia are presumed to be important stages in the development of gastric adenocarcinoma [16].

In our study, five cases of intestinal metaplasia were diagnosed. Of them, no cases showed positivity for H.pylori.

\section{Conclusion}

The incidence of Helicobacter Pylori was seen with chronic gastritis in our study. H. pylori are etiologically associated with chronic active gastritis, duodenal ulcer, gastric ulcer, primary gastric B-cell lymphoma or mucosal associated lymphoid type lymphoma (MALT lymphoma) and gastric adenocarcinoma.
H. pylori induced chronic gastritis were encountered in $16(47.22 \%)$ cases of the study. Overnight Giemsa stain was done on all the patients and a histopathological diagnosis was made along with the study of the incidence of $\mathrm{H}$. pylori positivity in all these patients.

However, As It is studied on a small number of cases for the smaller period so evidence may be very accurate if a study is conducted on a large number of sample for a larger period. The exact incidence judged in an accurate way.

\section{Funding: Nil, Conflict of interest: None. Permission of IRB: Yes}

\section{References}

1. Vijaya D et al, Simple stain for Helicobacter Pylori. Journal of Clinical and Diagnostic Research,2012 May (suppl-2),Vol-6(4):664-666

2. Harrison's Principle of internal Medicine $17^{\text {th }}$ Edition. Helicobacter Pylori infections, Page no.946

3. Steven J.Czinn. Helicobacter Pylori infection: Detection, Investigation, Management. The Journal of Pediatrics March 2005

4. Robbins and Cotran Pathologic Basis of Disease $8^{\text {th }}$ Edition, The Gastrointestinal TractPage no 778.

5. Sandhya Panjeta Gulia, Madhusudan Chaudhury, Naseem Noorunnisa, CD Balakrishnan, K. Balagurunathan: Interpretation of Upper Gastro-intestinal tract Endoscopic Mucosal Biopsies - A Study Conducted in Teaching Hospital In Puducherry, India. International Journal of Medical and Health Sciences July 2012,Vol1; Issue-3,17-24(ISSN 2277-4504)

6. Mustapha SK, Bolori MT, Ajayi NA, Nggadda HA, Pindiga UH, Gashau Wetal. Endoscopic finding sand the frequencyof Helicobacterpyloriamongdyspeptic patients in northeast tern Nigeria. The internet journal of gastro enterology 2007;6: 1 .

7. Katelaris PH, Tippet GH, Norbu P. Dyspepsia, Helicobacter Pylori, and peptic ulcer in a randomly selected population in India. Gut 1992; 33: 14621466 .

8. Mostaghni AA, AfaridM, Eghbali Sand, Kumar P. Evaluation of brushing cytology in the diagnosis of Helicobacterpy lorigastritis. Acta Cytol 2008; 52:597601. 


\section{Original Research Article}

9. Kalebi A, Rana F, Mwanda W, Lule G, Hale M. Histopathological profile of gastritis in adult patients seen at a referral hospital in Kenya. World $J$ Gastroenterol. 2007 Aug 14;13(30):4117-21.

10. Wyatt JI, Rathbone BJ. Immune response of the gastric mucosa to Campylobacter pylori. Scand J Gastroenterol Suppl.1988;142:44-9.

11. Marshall BJ, Warren JR. Unidentified curved bacilli in the stomach of patients with gastritis and peptic ulceration. Lancet. 1984 Jun 16;1(8390):1311-5.

12. Misra V, Misra SP, Singh MK, Singh PA, Dwivedi M. Prevalence of $\mathrm{H}$. pylori in patients with gastric cancer. Indian J PatholMicrobiol. 2007 Oct;50(4):702-7.
13. Eidt S, Stolte M. Prevalence of lymphoid follicles and aggregates in Helicobacter pylori gastritis in antral and body mucosa. J ClinPathol. 1993 Sep;46(9):832-5.

14. Genta RM, Hamner HW. The significance of lymphoid follicles in the interpretation of gastric biopsy specimens. Arch Pathol Lab Med. 1994 Jul;118(7): 740-3.

15. Chen XY, Liu WZ, Shi Y, Zhang DZ, Xiao SD, Tytgat GNJ. Helicobacter pylori-associated gastric diseases and lymphoid tissue hyperplasia in the gastric antral mucosa. J ClinPathol2002;55:133-7.

16. Geller SA. Small Organism, Many Challenges. Hum Pathol 1996;27(1):1-4.

\section{How to cite this article?}

Kumar V, Nag B. P, Anand P, Joshi N, Jain R. Incidence of Helicobacter Pylori in upper gastrointestinal lesion. Int J Med Res Rev 2018;6(01):43-48. doi:10.17511/ijmrr. 2018.i01.07. 